\title{
Genetics Evaluation Guidelines for the Etiologic Diagnosis of Congenital Hearing Loss
}

\author{
Genetic Evaluation of Congenital Hearing Loss Expert Panel
}

\begin{abstract}
The advent of hearing screening in newborns in many states has led to an increase in the use of genetic testing and related genetic services in the follow-up of infants with hearing loss. A significant proportion of those with congenital hearing loss have genetic etiologies underlying their hearing loss. To ensure that those identified with congenital hearing loss receive the genetic services appropriate to their conditions, the Maternal and Child Health Bureau of the Health Resources and Services Administration funded the American College of Medical Genetics to convene an expert panel to develop guidelines for the genetic evaluation of congential hearing loss. After a brief overview of the current knowledge of hearing loss, newborn screening, and newborn hearing screening, we provide an overview of genetic services and a guideline that describes how best to ensure that patients receive appropriate genetic services. The significant contribution of genetic factors to these conditions combined with the rapid evolution of knowledge about the genetics of these conditions overlaid with the inherently multidisciplinary nature of genetic services provides an example of a condition for which a well-integrated multidisciplinary approach to care is clearly needed. Genet Med 2002:4(3):162-171.
\end{abstract}

Key Words: newborn screening, genetic testing, congenital hearing loss

\section{BACKGROUND}

\section{Epidemiology}

Hearing loss is relatively common in the human population. Profound congenital hearing loss is estimated to occur in about 1 in 1000 births; approximately 50\% of cases are thought to be due to environmental factors and the remainder to genetic causes ${ }^{1,2}$ (Fig. 1). Examples of the former include acoustic trauma, ototoxic drug exposure (i.e., aminoglycosides), and bacterial or viral infections such as rubella or cytomegalovirus (CMV). Approximately $70 \%$ of congenital cases associated with genetic factors are classified as nonsyndromic (the deafness is not associated with other clinical findings that define a recognized syndrome). In the remaining 30\%, one of more than 400 forms of syndromic deafness can be diagnosed because of associated clinical findings. ${ }^{1,3}$ The auditory pathology varies widely among the many forms of syndromic hearing loss and includes both conductive and sensorineural deficits that may be unilateral or bilateral, symmetrical or asymmetrical, and progressive or stable. ${ }^{4}$

The auditory pathology of nonsyndromic hearing impairment (NSHI) can also vary, but the deficits are most often sensorineural. Congenital NSHI is usually subdivided by mode of inheritance: approximately $77 \%$ of NSHI is autosomal recessive, $22 \%$ is autosomal dominant, and $1 \%$ is X-linked. The associated "DeaFNess" gene loci are designated DFNB (autosomal recessive), DFNA (autosomal dominant), and DFN (X-linked). A variable proportion of NSHI, perhaps less than $1 \%$, is due to mitochondrial inheritance, ${ }^{2}$ but the proportion may be much higher $(10 \%-$

American College of Medical Genetics, 9650 Rockville Pike, Bethesda, MD 20814-3998. Go to www.geneticsinmedicine.org for a printable copy of this document.
$20 \%$ ) in some populations ${ }^{5,6}$ (Fig. 1). As a general rule, individuals with autosomal recessive NSHI have profound prelingual deafness, while dominant mutations lead to a more variable phenotype. More than $90 \%$ of children with congenital profound autosomal recessive NSHI are born to parents with normal hearing, while the remaining $10 \%$ or less are born to deaf parents.

Over the past 5 years, remarkable progress has been made identifying new hearing impairment loci and cloning new genes for deafness. To date, at least 77 loci for NSHI have been mapped: 40 autosomal dominant, 30 autosomal recessive, and 7 X-linked. ${ }^{7}$ As of July 2001, 50 auditory genes have been identified and sequenced including 14 for autosomal dominant disorders, 9 for autosomal recessive, 2 for X-linked, 5 mitochondrial, and at least 31 genes for syndromic hearing loss. In some cases, different mutations at the same locus have been found to cause syndromic and nonsyndromic forms of deafness. Although significant advances have been made, it is clear that more genes and mutations await discovery. Information about these genes and their protein products is revolutionizing our knowledge of the molecular processes involved in hearing and enhancing our understanding of how the alteration of these processes can lead to hearing loss. This knowledge may lead to mutation-specific therapies that can delay or prevent certain forms of genetic deafness such as the avoidance of aminoglycoside therapy in those with specific mitochondrial mutations.

\section{History of newborn screening programs}

Newborn screening programs for heritable disorders began in the early 1960s. ${ }^{8}$ They have evolved into the current public health newborn screening systems that include screening for metabolic diseases, hemoglobinopathies, endocrine disorders, cystic fibro- 


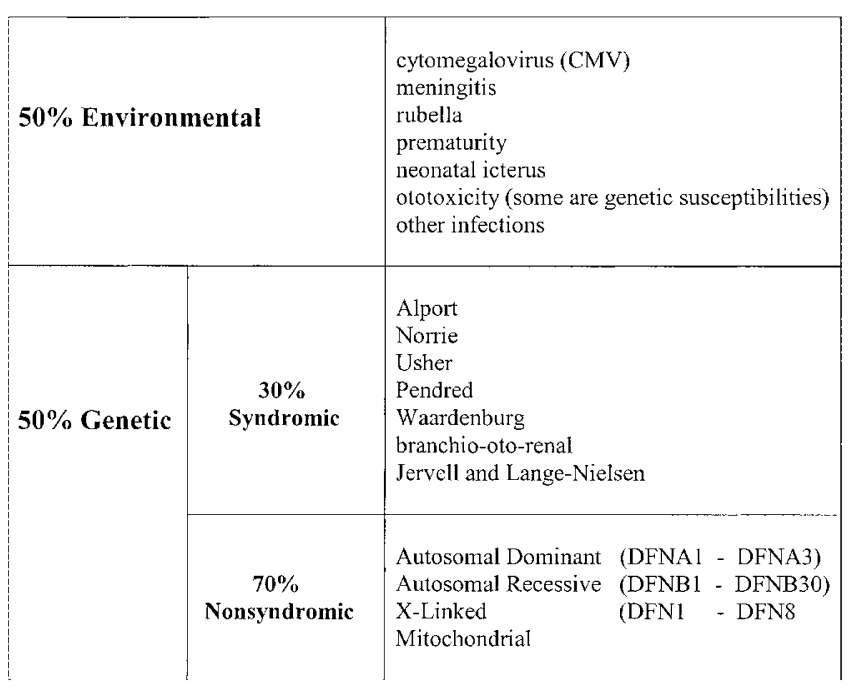

Fig. 1 "Distribution of causes" for profound hearing loss in infancy. The etiology of lesser degrees of hearing loss in the newborn period is not well understood.

sis, and infectious diseases. More recently, hearing loss has been added to this list of disorders amenable to newborn screening. Six essential components have been fundamental to the success of these programs: (1) screening in the newborn period; (2) prompt follow-up and referral; (3) diagnostic evaluation of infants with positive screening test results; (4) prompt planning and implementation of management strategies; (5) continuous program evaluation to ensure valid testing procedures, assess efficiency of follow-up and intervention, and evaluate benefits to the patient, family, and society; and (6) education of professionals and consumers about the benefits and procedures involved in newborn screening systems. In well-coordinated programs these components are integrated to achieve the fundamental goal of reduced mortality, morbidity, and disability for the screened infant.

In recent years, the impact of new technology and knowledge has caused a rapid expansion in the number of conditions for which newborn screening can be considered. ${ }^{9}$ These factors have led to considerable variation among states, with some states screening in either mandated or pilot programs for as few as three conditions and others for as many as 30 or more. ${ }^{10,11}$ In terms of the frequency of positive tests and the potential number of detectable causes, introduction of population-based screening for hearing loss represents a vast increase in the number of fundamentally different genetic and environmental conditions for which newborn screening is now performed. This is an important consideration that is not widely recognized. One of the goals of this report is to highlight some of the unique features of congenital hearing loss detectable through newborn hearing screening.

\section{History of hearing screening programs}

Since the mid-1960s, active multidisciplinary efforts have been directed to achieving early detection, diagnosis, and intervention for children who are deaf or hard of hearing. ${ }^{12}$ While individual hospitals began to screen newborns for hear- ing loss as early as 1970, widespread acceptance of this practice was not achieved until the late 1990s, following the $1993 \mathrm{NIH}$ Consensus Development Conference on Early Identification of Hearing Impairment in Infants and Young Children that recommended that all newborn infants be screened for hearing shortly after birth. ${ }^{13}$

Many factors have contributed to the recent adoption and spread of newborn screening for hearing loss. ${ }^{14,15}$ After an exhaustive evaluation of the relevant data, multiple professional societies, advocacy groups, and government agencies participating in the Joint Committee on Infant Hearing (JCIH $)^{16}$ endorsed universal newborn hearing screening as an essential component of early detection and intervention for infants with hearing loss. ${ }^{17}$ Professional organizations adopting the JCIH statement include:

- American Academy of Audiology

- American Academy of Pediatrics

- American Speech-Language-Hearing Association

- Council on the Education of the Deaf

- Directors of Speech and Hearing Programs in State and Health Welfare Agencies

Technologic advances in the physiologic evaluation of hearing in newborn infants have resulted in more accurate objective audiologic diagnosis, which has opened the door for earlier detection. The subsequent development of automated audiologic screening algorithms has made it possible to generate immediate outcomes from screening that do not require professional interpretation, thus facilitating the development of cost-efficient large-scale screening programs. The use of sensitive new technologies for screening (e.g., evoked otoacoustic emissions or auditory brainstem response testing) has substantially reduced the number of infants without hearing loss who fail the screening test (false positives) while achieving a high rate of detection for infants with a hearing loss (true positives), as shown by research funded by the National Institute on Deafness and Other Communication Disorders. ${ }^{18}$

Yoshinaga-Itano et al. ${ }^{19}$ and Moeller et al. ${ }^{20}$ have presented critically needed outcome data showing that early detection of hearing loss, accompanied by the introduction of intervention strategies within the first few months of life, results in infants' subsequent development of language skills that approach the skills of their peers who do not have hearing loss.

As of July 2001, more than two thirds of all states have enacted legislation establishing early hearing detection and intervention (EHDI) programs. Legislation is pending in most of the remaining states. Some states have established voluntary universal newborn hearing screening and intervention programs. The net impact is that $65 \%$ of all newborns are now screened for hearing loss shortly after birth, resulting in identification of two to three babies per 1000 births with permanent hearing loss (defined as 30 decibels or more in at least one ear). ${ }^{21}$ Increased participation in screening and improved coordination of the reporting of results of evaluations among providers and the medical home should enhance the benefits realized from newborn hearing screening. 
In 1999, landmark legislation enacted by the federal government provided funding to states from the Health Resources and Services Administration and the Centers for Disease Control and Prevention to support the infrastructure required for developing successful systems. This legislation included money for planning, development, implementation, and refinement of EHDI programs and for EHDI tracking and data management systems. The establishment of a large number of new programs provides an unparalleled opportunity to identify and collect relevant data on hearing-impaired infants who are detected by these programs. Analysis of these data will offer the maximum possible insight into secular and geographic variation in the etiology of hearing loss while also providing insight into the efficacy of early intervention in improving the communication skills acquired by hearing-impaired children.

\section{GENETIC EVALUATION PROGRAM}

\section{Genetic services overview}

The successful mapping of the human genome has created an explosion of clinically relevant knowledge that continues to expand as the functions of our 30,000 or more genes are identified. Geneticists can play a major role in the management of infants with newly detected hearing loss by facilitating the establishment of an etiologic diagnosis. The recently established statewide EHDI programs provide an unusual opportunity to develop cost-effective programs for delivering genetic services to this new client group. Because of the limited pool of professionals adequately trained to perform competent genetic evaluation and counseling for the families of deaf and hard-ofhearing infants, geneticists should not become involved in the evaluation of deaf and hard-of-hearing infants until the presence of a hearing loss has been confirmed. We strongly believe that a clearly articulated goal of EHDI programs should be the identification and inclusion of a competent clinical geneticist in the health care team managing newborns and infants with hearing loss.

In general, deafness differs from many other "complex" genetic diseases in that the hearing loss usually results from abnormalities in single genes or gene pairs. However, some forms of deafness are beginning to be recognized in which the hearing loss may result from the combined effects of genes at two or more loci. While abnormalities in many different single genes or gene pairs can cause deafness, a remarkable finding is that defects in a single gene, called GJB2, give rise to more than half of the genetic cases of profound deafness in the United States. The GJB2 gene codes for a gap junction protein of the beta subunit class named connexin 26 that is involved in cell-to-cell diffusion and recycling of small molecules such as potassium. DNA-based molecular genetic tests are being developed to accurately detect this and many other forms of genetic deafness. Unfortunately, the knowledge base about the complexity of genetic test results is still incomplete. Thus professionals who are fully aware of the implications and limitations of the methods, technology, and competencies of the laboratory should be the ones to interpret the test results, especially since many test results are straightforward, but others are not. The ability to understand and responsibly convey uncertainty is an inherent part of the training and practice of clinical geneticists, and only if an adequate system for the interpretation of genetic test results is available can their routine use for the evaluation of children with deafness be recommended. Infants and children with lesser degrees of hearing loss also need evaluation by a clinical dysmorphologist because little is known about the causes of deafness in this group of infants and children, and some, perhaps many, may have subtle clinical abnormalities that would allow diagnosis of a specific form of syndromic deafness.

At the current time, interpretation of genetic testing results ranges from the less complex to the highly complex. The time between development of a new genetic test and its application for medical diagnosis can be quite short. As knowledge accumulates about the range of normal and pathologic variation, and new test procedures are developed, the responsible interpretation of genetic test results may become increasingly difficult. Complexity occurs because the most common and easily understood genetic variations are found first and the less frequent, more complex variations are only identified when more people from a greater variety of ethnic backgrounds are evaluated. In many cases, a genetic variant or a so-called "private mutation" may be confined to a single family, and the strict evidence that it is of etiologic significance may be limited to its cosegregation with a disease in that family and the fact that different mutations involving the same gene cause the disease in other families. For example, more than 45 different mutations in the gene GJB2 can cause autosomal recessive NSHI, but most individuals of Northern European heritage with GJB2 deafness carry at least one common mutation, the 35delG (deletion of a guanine nucleotide at position 35 from the start of the initiation codon). ${ }^{22}$ In cases in which the spectrum of mutations for any one gene may include common and rare variants, the usual requirements for establishing the clinical sensitivity and specificity for every possible mutation must be relaxed if the benefits of our investment in human genetics research are to be realized by the general public. The potential for variation in the human genome is so vast that the discovery of new mutations in disease-associated genes will be a neverending process. Obviously, the clinical use of mutation screening for diagnostic purposes cannot be delayed until every mutation has been discovered.

Over time, the interpretation of test results showing wellunderstood variations becomes the standard. However, given the almost unlimited capacity for DNA to vary among individuals, it is reasonable to expect that complex interpretive issues will always arise for other less common DNA changes. Therefore, genetic counseling can also range from the communication of basic, well-established facts to the complex interpretation of imperfect knowledge that requires special skills in the characterization and transmission of uncertainty. In some families, complex statistical analysis based on available clinical and pedigree data may be required to provide the most accurate characterization of genetic risks. Finally, genetic testing 
also may reveal unexpected results such as nonpaternity that may impact recurrence chances addressed in counseling. Hence, the provision of comprehensive genetic services is commonly a multidisciplinary effort involving professionals with special competence in clinical evaluation, laboratory testing, statistical analysis, and counseling.

It is not surprising that the issues that arise in providing competent evaluation and counseling of the families of deaf individuals range from relatively straightforward traits to some of the most complex problems and pedigrees encountered in human genetics. Reducing the incidence and/or morbidity associated with hearing loss requires:

- Improved recognition of the importance of genetic factors in the etiology of deafness. Specific examples include obstetricians' awareness of the importance of a family history of hearing loss as a potential risk factor for aminoglycoside ototoxicity in the neonatal intensive care unit population and communicating this information to neonatologists.

- Recognition that not all forms of hearing loss are expressed at birth and that surveillance, including the possible use of molecular tests to identify high-risk cases who would benefit from continued monitoring of their hearing, is appropriate.

- Early identification of patients with certain forms of syndromic deafness which can dramatically alter the risk of serious complications for the affected infant and other family members. Timely diagnosis of some genetic conditions such as biotinidase deficiency and aminoglycoside sensitivity can actually lead to prevention of hearing loss.

\section{Definition of the condition}

The definition of hearing loss used in this statement and in screening programs is consistent with that of the Joint Committee on Infant Hearing Year 2000 Position Statement and Guidelines. ${ }^{16}$ This loss is permanent and is bilateral or unilateral, sensory or conductive, and averages 30 decibels or more in the frequency region important for speech recognition.

\section{Evaluation}

Appropriate management of all persons identified with congenital hearing loss, as defined above, requires a comprehensive genetic evaluation. As noted previously, certain core personnel are required for the initial genetic evaluation and counseling. Depending on the clinical findings, other specialists may be required for a comprehensive evaluation. Core personnel include individuals with expertise in (1) the genetics of hearing loss, (2) dysmorphology, (3) audiology, (4) otolaryngology, (5) genetic counseling, and (6) communicating with parents who may themselves be deaf, including use of qualified interpreting services.

Based on the outcome of the evaluation, various other types of professional expertise may also be needed, especially with syndromal hearing loss (e.g., ophthalmology, cardiology, nephrology, neurology, etc.).
The evaluation should include, at a minimum, a family history targeted at key genetic and phenotypic features of the many forms of syndromic and nonsyndromic hearing loss, and a similarly targeted patient history and physical examination (see Appendix 1 for details). The purpose of the genetic evaluation is to establish an etiologic diagnosis.

The family history should specifically address:

1. Pedigree (three to four generations) with attention to consanguinity, paternity, and hearing status of the parents and siblings.

2. Ethnicity and country of origin.

3. Inheritance pattern of hearing loss ( $\mathrm{AD}, \mathrm{AR}, \mathrm{X}$-linked, mitochondrial).

4. Audiometric characteristics in any deaf and hearing-impaired family members (age of onset, progression, conductive hearing loss, nonsyndromic hearing loss, auditory neuropathy). (An attempt should be made to collect and review previous records.)

5. Evidence of vestibular dysfunction.

6. Syndromic versus nonsyndromic features.

To establish whether the loss may result from a specific syndrome, the practitioner should evaluate for, or inquire about, the following conditions in the patient and/or relatives:

1. Visual anomalies, including heterochromia irides, retinitis pigmentosa, myopia, retinal detachment, and early cataracts.

2. Facial/cervical dysmorphology, including synophrys, dystopia canthorum, preauricular pits, aural atresia, branchial cysts, cleft palate, and dental anomalies.

3. Endocrine abnormalities such as thyromegaly and diabetes.

4. Cardiac signs or symptoms, including syncope, sudden death, arrhythmia, prolonged QT interval, fainting spells, and congenital heart defect.

5. Renal abnormalities, including hematuria, proteinuria, and structural defects.

6. Integumentary changes such as premature graying, white forelock, abnormal pigmentation, or dry skin/keratoderma.

The patient history should include specific risk factors such as:

1. Intrauterine infections (e.g., toxoplasmosis, other agents, rubella, CMV, herpes simplex [TORCH]).

2. Meningitis.

3. Extracorporeal membrane oxygenation (ECMO).

4. History of hypoxia.

5. Prenatal alcohol exposure.

6. Exposure to ototoxic drugs.

The physical examination should include:

1. Otologic examination.

2. Airway examination.

3. Documentation of dysmorphisms. 
4. Neurologic evaluation, including assessment of vestibular function in older patients.

After obtaining a family history, patient history, and physical examination, it may be possible to ascribe an etiology for the hearing loss. For example, based on the family history and physical examination, a diagnosis of branchio-oto-renal (BOR) syndrome may be made. However, in about $30 \%$ of patients, no obvious etiology will be apparent. It is important to emphasize that a genetic cause is not excluded by such an evaluation because patients with recessive NSHI can present without a family history of hearing loss (i.e., as a simplex case). An important goal of genetic evaluation is to attempt to distinguish these isolated or simplex cases in which there may be a $25 \%$ chance of deafness in subsequent offspring from sporadic cases caused by environmental factors with a low chance of recurrence in families with no history of hearing loss.

\section{Triage/testing and triage paradigm}

The goal of triage/testing is to establish an etiologic basis for hearing loss in the most efficient manner possible. Based on results of the genetic evaluation, the following should be considered:

1. If a form of syndromic deafness is suspected:

- Gene-specific mutation screening can be obtained in many cases and more tests will undoubtedly become available.

2. If nonsyndromic deafness is suspected AND the patient is a simplex case:

- CMV testing should be performed. A negative test for CMV antibodies in early infancy may exclude CMV-related hearing loss. A positive result must be interpreted with caution.

- GJB2 (connexin 26) mutation screening should be obtained by sequence analysis. A negative test result does not exclude a genetic etiology; a positive test result may make it possible to avoid other expensive and potentially invasive tests.

3. If nonsyndromic deafness is suspected AND the patient is a multiplex case with other hearing-impaired first-degree relatives, proceed directly to connexin 26 testing.

4. If nonsyndromic deafness is suspected AND the pedigree suggests dominant inheritance, connexin-related deafness is not excluded and gene-specific mutation screening for other loci may be available on a research or clinical investigation basis and will no doubt become increasingly more available.

5. If nonsyndromic deafness is suspected AND the pedigree suggests mitochondrial DNA inheritance:

- Testing for the A1555G mutation (associated with aminoglycoside-induced hearing loss) and the A7445G mutation, both of which are associated with some rare familial cases of hearing loss, may be appropriate after common GJB2 mutations are excluded.

6. If nonsyndromic deafness is suspected AND both parents are deaf:

- Connexin-related deafness will be strongly suspected. (Because mutations in GJB2 are the most common cause of deafness in the United States, the vast majority of marriages between deaf individuals who produce deaf offspring are between individuals with GJB2 mutations.)

- After triage/testing, it will be possible to ascribe a genetic etiology to the hearing loss in many persons. For example, a child may be diagnosed with GJB2-related deafness. If two clearly pathologic alleles are found, we then know the cause of that child's deafness with virtual certainty and can accurately predict the chance of recurrence in a subsequent child.

- Alternatively, mutation screening may be negative. A negative mutation screen must NOT be taken to mean that the deafness is NOT genetic. This distinction is subtle but important, and currently is not adequately conveyed to parents of deaf children who undergo genetic testing. ${ }^{23} \mathrm{In}$ patients with an unremarkable family history of deafness and a negative test result for connexin 26 , the probability that the deafness is genetic can be given. The probability will vary based on the number of hearing siblings. The proportional distribution of the different mutations in the connexin 26 locus also varies in different ethnic groups. ${ }^{22}$

\section{Counseling and education}

The purpose of genetic counseling is to ensure that the parents/patients understand the findings and limitations inherent in any genetic evaluation. Successful delivery of genetic services to parents/families requires that professionals be sensitive to cultural orientation and communication and psychosocial needs. ${ }^{24}$ An individual's approach to health may be based on ethnicity, cultural hearing status, socioeconomic status, etc. At least $10 \%$ of deaf infants will have deaf parents who may consider themselves to be culturally Deaf. These infants represent an important group identified by newborn hearing screening programs. Service providers should ensure that arrangements for professional sign language or oral interpreters are made for deaf or hard-of-hearing parents. As with other cultures, members are characterized by unique social and societal attributes and a unique language (American Sign Language or ASL). It is important in genetic counseling situations that family members not be used as interpreters, since well-meaning family members may filter information and have their own emotional stake in the information being shared.

Optimally, counseling for deafness includes both pretest and posttest sessions. A pretest session focuses on factual information, including an explanation of the different causes of deafness, the types of genetic deafness that might play a role in a specific family, modes of inheritance, and genetic testing options, including their risks, benefits, and limitations. A posttest 
session includes an explanation of test results in the context of the pretest session and an assessment of the psychosocial impact that the results may have on the parents, child, and family. It also is important to obtain feedback from families after providing genetic testing to assess their understanding of the impact of these data.

Some deaf individuals have expressed reluctance to participate in genetic counseling for fear that they will be told not to have children. ${ }^{25}$ However, some of these couples may welcome the birth of a deaf child. ${ }^{25}$ Information and services should be provided in a fashion that is sensitive to different reproductive preferences or cultural differences. For example, during genetic counseling, word choice can convey cultural bias. Use of the words "chance" instead of "risk" for having a deaf or hearing child and "deaf or hearing" instead of "affected or unaffected" can help in removing cultural bias. ${ }^{24}$ Other terms such as "handicapped," "pathology," "impairment," may be offensive or inappropriate to deaf and hard-of-hearing adults and to hearing parents as well.

Receptivity of parents to etiologic information may be profoundly influenced by their emotional reaction to the diagnosis of hearing loss in their child. Hearing parents may experience grief and confusion regarding this unexpected diagnosis and may feel a sense of urgency about where to turn for information and decisions about communication and treatment options. It is important for professionals to know about appropriate referral networks specific to hearing loss for emotional and decision-making support as referral of parents to support services such as parent groups or psychosocial counseling is appropriate in many situations. (See Appendix 2, Educational and Informational Resources. Note that many resources are available and this list is not meant to be exclusionary or biased.)

It is important to empower parents by providing information and support in order to help them avoid overdependence on professionals and to involve them in decisions regarding habilitation, etc. Parents may be more receptive to etiologic information after their psychosocial needs have been met. In addition to ensuring that medical needs are met, etiologic information can be extremely helpful in relieving guilt that parents may feel about having a child with hearing loss (e.g., a mother who may blame herself for the hearing loss due to an unrelated event during her pregnancy) and can allay worry about other complications that may appear in their child.

Attitudes of hearing parents and deaf and hard-of-hearing people toward genetic testing for deafness are also important to consider, although such attitudes may not be predictive of behavior. Several studies of attitudes have been completed ${ }^{23,26,27}$ and demonstrate a variety of opinions about the appropriate use of genetic technologies. While some deaf and hearing people had a positive attitude toward genetic technology, many were opposed to the use of genetic testing during prenatal diagnosis for the purpose of pregnancy termination based on the hearing status of the fetus. These surveys point out the need for further studies to determine the utilization patterns of prenatal diagnosis for hearing loss and the need for all stakeholders to engage in a discussion regarding the appropriate use of such techniques.

Given the heightened public awareness of genetics, many people have the incorrect belief that their DNA defines them. Genetic information has even been called an individual's "future diary." 28 It is hardly surprising that one's self-perception can be altered by learning that one has a genetic condition or is a carrier for a genetic mutation. ${ }^{29}$ Furthermore, a unique characteristic of genetic tests and information that separates them from other medical information is that they reveal facts about the entire family. Whereas most medical information applies only to the individual seeking treatment, genetic information has implications for anyone who shares or may share genes with the person being tested. Obvious implications arise regarding genetic privacy for relatives of the patient. Do these persons have a right to this information, even against the wishes of the person who was tested? Conventional medical ethics says no, as test results are privileged information to be shared only with the expressed consent of the patient or the patient's parents. ${ }^{30,31}$ However, some ethicists have argued that all biologically related individuals have some claim to this information. As genetic testing becomes more common, the scope of this problem will grow and will need to be addressed. To date, neither the courts nor the legislative bodies have provided a definitive standard of practice on this issue.

Issues of genetic discrimination may also arise. While there is much legislation to protect against it, insurance discrimination and even loss of insurance are a common concern for people undergoing genetic testing.

\section{Habilitation}

Various treatment/habilitation options (e.g., otologic, audiologic, linguistic, and ophthalmologic) are available and may be considered. Parents should be made aware of the available options as part of the counseling process.

\section{Follow-up}

The purpose of follow-up is to ensure that the continued health and hearing/aural needs of the patient and family are being met. Follow-up should specifically include:

- Repeating the genetic evaluation, particularly if a specific diagnosis was not made initially or if test results or additional molecular tests have become available.

- Repeating the audiometric evaluation.

- Evaluation of progressive loss. (An imaging study should be obtained. The finding of Mondini dysplasia or dilated vestibular aqueduct should prompt mutation screening of SLC26A4 for Pendred syndrome/DFNB4.)

- Repeating the otolaryngologic examination.

- Other requests for subspecialty-directed examinations based on the particular patient's needs and associated clinical findings. For example, patients at risk for neurofibromatosis type 2 or suspected of having Stickler syndrome or Usher syndrome may require ophthalmologic evaluation. Families with history of syncope or sudden 
death may require cardiac evaluation, while those with evidence of renal disease may require a nephrology evaluation to exclude Alport syndrome or BOR syndrome.

\section{BENEFITS}

A number of benefits can result from ensuring an appropriate genetic evaluation as described in these guidelines. It is possible to avoid unnecessary, costly clinical tests such as electroretinograms, temporal bone imaging, and electrocardiograms. Determining or defining the cause of the hearing loss provides an etiologic diagnosis that can dispel misinformation and offer emotional support by allaying parental guilt. Etiologic diagnosis also provides the basis for determining the chance for deafness and recurrence of deafness within families. (For example, a person with autosomal recessive GJB2-related deafness and his/her hearing spouse have a 50\% chance of having child with hearing loss if the hearing spouse also carries a recessive GJB2 deaf-causing allele. Alternatively, if the hearing spouse carries two normal GJB2 alleles, the chance of having a deaf child is less than $10 \%$ and varies with the number of hearing siblings.) Knowledge of etiology allows one to anticipate potential/associated health problems and to offer appropriate referral for therapeutic options (e.g., monitoring for myopia and early retinal detachment in Stickler syndrome; obtaining a renal examination to identify kidney complications in BOR) and medical interventions such as amplification implants.

An individualized/personalized health maintenance strategy may be implemented. For example, vitamin A therapy may be beneficial to persons with Usher syndrome in slowing retinal degeneration, and treatment of children with Jervell and Lange-Nielsen syndrome can minimize cardiac complications. Diagnosis of biotinidase deficiency in children identified in newborn screening programs enables biotin treatment to avoid hearing loss. In persons carrying the mitochondrial mutation, mtDNA A1555G, avoidance of aminoglycoside antibiotics reduces the risk of developing hearing loss.

\section{RESEARCH NEEDS/ANTICIPATING CHANGES}

The evaluation of scientific and medical literature on which these recommendations are based also revealed a number of areas in which data are deficient and research is needed. Similarly, the history and speed of genetic test development allows us to anticipate changes likely to be evident in the near future. Expanding knowledge of human genetic or infectious causes of later-onset congenital hearing loss offers the potential for the addition of more genetic tests into newborn hearing screening programs. Rapidly evolving new technologies will likely allow the addition of microarrays and other kinds of high-throughput testing to supplement the early detection of hearing loss using available resources (e.g., newborn screening residual dried blood spots) and testing for a broad spectrum of deafness and hearing loss-causing genes.

As individuals with genetic causes of deafness are identified, the opportunity to determine genotype/phenotype correla- tions will emerge. Simultaneously, with the identification of families with inherited types of hearing loss, the opportunity to understand better the variability in the expression of the mutations will be possible. Knowledge of the biological pathways in which the gene products are involved will likely allow for a better understanding of this variable expressivity. The development and evaluation of gene-specific therapy (e.g., cost benefit of vitamin A, antiusherin antibiotics, or medication to reduce retinitis pigmentosa sequelae) will be possible. There will also be opportunities to develop and test new clinical tools such as a simple tool for vestibular assessments of infants.

The continued integration of genetics into public health will require that there be a broader understanding and appreciation of individual and population variability. The genetic deafness load is population/ethnic-specific with most data derived from populations of Northern European extraction. Data applicable to a broad range of ethnic groups is required to ensure comparable clinical sensitivity of mutation detection in all ethnic groups. It will also be necessary to develop and implement consistent data management tools among various newborn screening programs. With the rapid introduction of molecular methods into both hearing and classical newborn screening programs, such tools will allow for uniform communication, evaluation, and comparison of screening programs and strategies to identify the most successful screening and follow-up programs.

\section{LIMITATIONS}

A number of limitations exist with regard to the delivery of the genetic services related to congenital hearing impairment. An underlying problem that these guidelines seek to minimize is the limited education, exposure, and experience with genetics and genetic services of most in the health care system. Few people are trained in delineating the etiology of these disorders, thereby complicating implementation of a complete program in all areas of the country. Model systems must be developed and disseminated. Because of the high volume of testing expected in this area, it is important that attention and support be given to the development of links among the many providers involved in the identification, diagnosis, and follow-up of congenital hearing loss. Further exacerbating the limited knowledge of genetics by many providers is the rapidly developing and, therefore, unstable body of knowledge of the genotype/phenotype correlations and the expanding technologies used to detect the mutations associated with these conditions. This rapid development of the knowledge base and new tests also requires us to consider issues related to recontacting patients and ensuring that patients maintain links to providers.

Many genetic tests for hereditary hearing loss remain quite variable in methods and mutations tested. Clinical sensitivity is often less than $100 \%$, and patients must appreciate that not identifying one of the known mutations does not necessarily mean that one is not present. The genetics provider community must work toward standardizing testing. In addition, only a limited number of laboratories offer testing related to the 
conditions in question. Data about the natural history of the development of hearing loss are also quite limited for many of its causes.

To ensure the maximal data acquisition to improve and finetune our understanding of clinical validity and utility of these tests, data collection that is currently ad hoc should be well coordinated and centralized to maximize the value of its content.

\section{GENERAL INFORMATION AND EDUCATION RESOURCES}

Numerous resources are available to professionals and to the public. See Appendix 2, Educational and Informational Resources, for a partial list that includes those that offer comprehensive lists of available resources.

\section{Approved by the Board of Directors of the American College of Medical Genetics, October 2001. (C) AMERICAN COLLEGE OF MEDICAL GENETICS, 2002.}

\section{Acknowledgments}

This report was produced through the support of the Maternal and Child Health Bureau, Health Resources and Services Administration (HRSA) Contract 01-MCHB-25A to the American College of Medical Genetics. The authors also acknowledge those individuals serving on the Genetic Evaluation of Congenital Hearing Loss Expert Panel:

Walter E. Nance, Chair, Medical College of Virginia, Virginia Commonwealth University

Kathleen S. Arnos, Gallaudet University

John C. Carey, University of Utah Health Science Center

George C. Cunningham, California Department of Health

Rena Ellen Falk, Cedars-Sinai Medical Center

Terese Finitzo, OZ Systems

Dynio Honrubia, Harvard Medical School

Bronya J. Keats, Louisiana State University

William J. Kimberling, Boys Town National Research Hospital

Gail Lim, Pediatrix Medical Group

Cynthia C. Morton, Brigham and Women's Hospital, Harvard Medical School

Arti Pandya, Medical College of Virginia, Virginia Commonwealth University

Mary Kay Pelias, Louisiana State University

James Skordas, Pediatrix Medical Group

Richard Smith, University of Iowa Hospitals and Clinics

Michael S. Watson, Project Director, American College of

Medical Genetics, Washington University

Ex Officio:

Colleen Boyle, Centers for Disease Control and Prevention

Aileen Kenneson, Centers for Disease Control and Prevention

Amy Donahue, National Institute on Deafness and Other Communication Disorders, NIH

Michele Lloyd-Puryear, Maternal and Child Health Bureau, HRSA

Marie Mann, Maternal and Child Health Bureau, HRSA
Jay Sheehan, Maternal and Child Health Bureau, HRSA

Bradford Therrell, National Newborn Screening and Genetics Resource Center, University of Texas Health Science Center at San Antonio

Karl R. White, National Center for Hearing Assessment and Management

\section{References}

1. Gorlin RJ, Toriello HV, Cohen MM. Hereditary hearing loss and its syndromes. New York: Oxford University Press, 1995.

2. Morton NE. Genetic epidemiology of hearing impairment. Ann N Y Acad Sci 1991; 630:16-31.

3. Steel KP, Kros CJ. A genetic approach to understanding auditory function. Nat Genet 2001;27:143-149.

4. Resendes BL, Williamson RE, Morton CC. At the speed of sound: gene discovery in the auditory nervous system. Am J Hum Genet 2001;69:923-935.

5. Estivill X, Govea N, Barcelo A, Perello E, Badenas C, Romero E, Moral L, Scozzari R, D'Urbano L, Zeviani M, Torroni A. Familial progressive sensorineural deafness is mainly due to the mtA155G mutation and is enhanced by aminoglycosides. Am J Hum Genet 1998;62:27-35.

6. Pandya A, Xia XJ, Erdenetungalag R, Amendola M, Landa B, Radnaabazar J, Dangaasuren B, Van Tuyle G, Nance WW. Heterogeneous point mutations in the mitochondrial tRNA Ser (UCN) precursor coexisting with A1555G mutation in deaf students from Mongolia. Am J Hum Genet 1999;65:1803-1806.

7. Van Camp G, Smith RJH. Hereditary hearing loss homepage. 2001 http://dnalabwww.uia.ac.be/dnalab/hhh.

8. Newborn Screening Task Force. Serving the family from birth to the medical home: a report of the Newborn Screening Task Force convened in Washington DC, May 10-11, 1999. Pediatrics 2000;106:383-427.

9. Pass KA, Lane PA, Fernhoff PM, Hinton CF, Panny SR, Parks JS, Pelias MZ, Rhead WJ, Ross SI, Wethers DL, Elsas LJ. US newborn screening guidelines, II: follow-up of children, diagnosis, management, and evaluation. Statement of the Council of Regional Genetics Networks (CORN). J Pediatr 2000;137:S1-S46.

10. Therrell B. US newborn screening policy dilemmas for the 21 st century. Mol Genet Metab 2001;74:64-74.

11. NNSGRC (National Newborn Screening and Genetics Resource Center). http//www.Genes-R-Us.UTHSCSA.edu, 2001.

12. Downs MP, Sterritt GM. Identification audiometry for neonates: a preliminary report. J Audiol Res 1964;4:69-80.

13. NIH Consensus Statement. Early identification of hearing impairment in infants and young children. NIH Consens Statement 1993;11:1-24.

14. Joint Committee on Infant Hearing. Position statement. Pediatrics 1995; 1994:95: 152-156.

15. American Academy of Pediatrics Policy Statement. Newborn and infant hearing loss: detection and intervention. Pediatrics 1999;103:527-530.

16. Joint Committee on Infant Hearing Year 2000 Position Statement. Principles and guidelines for early hearing detection and intervention programs. $2000 \mathrm{http} / / \mathrm{ww}-$ w.audiology.org/professional/positions/jcih-early.php.

17. American College of Medical Genetics. Statement of the American College of Medical Genetics on universal newborn hearing screening. Genet Med 2000;2:149-150.

18. Norton SJ, Gorga MP, Widen JE, Folsom RC, Sininger Y, Cone-Wesson B, Vohr BR, Fletcher KA. Identification of neonatal hearing impairment: summary and recommendations. Ear Hear 2000;21:529-535.

19. Yoshinaga-Itano C, Sedey AL, Coulter DK, Mehl AL. Language of early- and lateridentified children with hearing loss. Pediatrics 1998;102:1161-1171.

20. Moeller MP. Early intervention and language development in children who are deaf and hard of hearing. Pediatrics 2000;106:1 www.pediatrics.org/cgi/content/full/106/ e43

21. NCHAM (National Center for Hearing Assessment and Management). 2001 http:// www.InfantHearing.org.

22. Kenneson A, Van Naarden Braun K, Boyle C. GJB2 (connexin 26) variants and nonsyndromic sensorineural hearing loss. Genet Med. In press.

23. Brunger JW, Murray GS, O’Riordan M, Matthews AL, Smith RJ, Robin NH. Parental attitudes toward genetic testing for pediatric deafness. Am J Hum Genet 2000;67: 1621-1625.

24. Israel J. An introduction to deafness: a manual for genetic counselors. Washington, DC: Gallaudet Research Institute, Gallaudet University, 1995.

25. Prezioso CT. Cultural aspects of deafness (the deaf community). In: Israel J, editor. An introduction to deafness: a manual for genetic counselors. Washington, DC: Gallaudet Research Institute, Gallaudet University, 1995:133-145.

26. Middleton A, Hewison J, Mueller RE. Attitudes of deaf adults toward genetic testing for hereditary deafness. Am J Hum Genet 1998;63:1175-1180. 
27. Middleton AM, Hewison J, Mueller R. Prenatal diagnosis for inherited deafness: what is the potential demand? J Genet Couns 2001;10:121-131.

28. Troy ESF. The genetic privacy act: an analysis of privacy and research concerns. J Law Med Ethics 1997;25:256-272.

29. Suter SM. Whose genes are these anyway? Familial conflicts over access to genetic information. Mich Law Rev 1993;91:1854-1908.

30. Miller H. DNA blueprints, personhood, and genetic privacy. Health Matrix 1998;8: $179-221$.

31. Green RM, Thomas AM. Whose gene is it? A case discussion about familial conflict over genetic testing for breast cancer. J Genet Couns 1997;6:245-254

\section{Appendix 1}

\section{Important Evaluation Considerations for Genetic Service Providers: Checklist items}

A. Family history components for determining etiology of congenital hearing loss

Ethnicity

Consanguinity

Family history of hearing loss: If yes, determine type, severity, age of onset, stability versus progression, presence or absence of vestibular symptoms, other associated findings, availability of records

Targeted family history by systems (three to four generations): Integumentary: white forelock (may repigment with time); other unusual hair color, texture, or pattern (e.g., sparse, unusually curly); premature gray hair; acquired or congenital, white or brown spots (macules) or lentigines; abnormal eyebrows, lashes, or nails; dry skin/keratoderma

HEENT: abnormal ear shape, size, ear canals, preauricular skin tag or sinus; ocular malformation, iris heterochromia, congenital glaucoma, cataract, moderate-severe myopia, retinal detachment, optic atrophy, or retinitis pigmentosa; cleft lip and/or palate; micrognathia, other craniofacial or ocular malformation or abnormality

Neck: sinus, cartilage remnant, cyst, mass, goiter

Cardiovascular: congenital malformation; irregular heart beat; arrhythmia; fainting spells; history of sudden infant death syndrome in a close family member

Gastrointestinal: Hirschsprung disease; severe chronic constipation beginning in early childhood

Skeletal: unusually tall or short stature, "marfanoid" habitus, scoliosis, joint laxity, any limb anomaly

Neurologic: child or fetus with neural tube defect; any progressive vision loss, ptosis, nystagmus; seizures, especially associated with sudden death; muscle weakness, myopathy, muscular dystrophy, myotonia; chronic or progressive ataxia; other progressive or debilitating neurologic disorder

Endocrine: diabetes mellitus, diabetes insipidus; goiter, other thyroid disease; hypogonadism, infertility

B. Congenital hearing loss: prenatal and perinatal history

Prenatal history

Maternal fever (document magnitude and duration)

Maternal rash (document type, location, duration)

Maternal known exposure

Maternal TORCH status

Viral cultures or polymerase chain reaction studies at birth
Other evidence of prenatal infection

Prenatal exposure to potentially ototoxic drugs including alcohol and cocaine

Hydrops fetalis

Perinatal history

Intrauterine growth restriction

Low birth weight appropriate for gestational age $(<1500 \mathrm{~g})$

Low Apgar score(s)

One-minute Apgar 0-4

Five-minute Apgar 0-6

Neonatal intensive care unit admission

Neonatal meningitis

Mechanical hyperventilation (with alkalosis)

Persistent hypotonia (at least $>2$ hours)

Neonatal seizures

Hyperbilirubinemia requiring exchange transfusion (or meeting criteria for exchange)

Persistent pulmonary hypertension/fetal circulation; ECMO

Treatment with aminoglycoside antibiotics

Failed newborn screening for hypothyroidism

Documentation of prior evaluations

TORCH titers, especially IgM for rubella and CMV

Electrocardiogram, especially prolonged monitoring CMV testing by culture or DNA in the neonatal period Computed tomography or magnetic resonance imaging of the temporal bone: Mondini defect; dilated vestibular aqueduct

Mutation analysis for a known deafness gene

Ophthalmology consult

\section{Appendix 2}

\section{Educational and Informational Resources}

The majority of resources listed below are comprehensive compilations of resources developed by national organizations and federal agencies. Additional important Internet-accessible resources of research and practice databases are included.

- Communicating Informed Consent to Individuals Who Are Deaf and Hard-of-Hearing. National Institute on Deafness and Other Communication Disorders (NIDCD)

- NIH Publication 00-4689, September 1999

- Directory of Information Resources for Human Communication Disorders. National Institute on Deafness and Other Communication Disorders (NIDCD) Information Clearinghouse, 1 Communication Avenue, Bethesda, MD 20892

- Early Identification of Hearing Loss: Implementing Universal Newborn Screening Programs. National Maternal and Child Health Clearinghouse, 2070 Chain Bridge Road, Suite 450, Vienna, VA 22182-2536

Web sites for the public

- Early Hearing Detection and Intervention Program (EHDI): www.cdc.gov/ncbddd/ehdi 
- National Center for Hearing Assessment and Management (NCHAM): www.infanthearing.org

Web site for hereditary hearing loss researchers

- Hereditary hearing loss: www.uis.ac.be/dnalab/hhh
Web sites for professionals

- GeneClinics: www.geneclinics.org

- National Newborn Screening Genetics Resource Center: Genes-R-Us.UTHSCSA.edu

This guideline is designed primarily as an educational resource for medical geneticists and other health care providers to help them provide quality medical genetic services. Adherence to this guideline does not necessarily ensure a successful medical outcome. This guideline should not be considered inclusive of all proper procedures and tests or exclusive of other procedures and tests that are reasonably directed to obtaining the same results. In determining the propriety of any specific procedure or test, the geneticist should apply his or her own professional judgment to the specific clinical circumstances presented by the individual patient or specimen. It may be prudent, however, to document in the patient's record the rationale for any significant deviation from this guideline. 